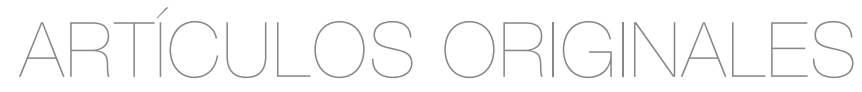

\title{
Tendencias de la taxonomía de generaciones en elámbito laboral: un análisis bibliométrico
}

pp. 113-126

\author{
RICARDO CARLOS ALVARADO ALFARO* \\ ERICK LEOBARDO ÁLVAREZ-AROS**
}

* Doctorando en Dirección de Organizaciones. Universidad Popular Autónoma del Estado de Puebla, Puebla, México. E-mail: ricardocarlos.alvarado@upaep.edu.mx.ORCID: 0000-0003-1629-0025.GoogleScholar: https://scholar.google.com/citations?user=ooFptwAAAAJ\&hl=es.

** Posdoctorado en Innovación Abierta y Competitividad Tecnológica. Universidad Popular Autónoma del Estado de Puebla, Puebla, México. E-mail: erickleobardo.alvarez@upaep.mx. ORCID: 00oo-0002-1934-5442. Google Scholar: https://scholar.google.com/ citations?hl=es\&user=URVSTXYAAAAJ. 


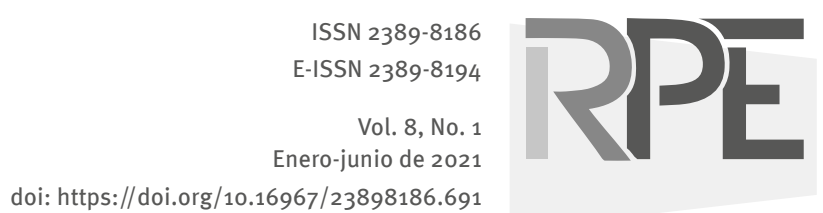

COMO CITAR ESTE ARTÍCULO

How to cite this article:

Alvarado, R. y Álvarez-Aros, E. (2021). Tendencias de la taxonomía de generaciones en el ámbito laboral: un análisis bibliométrico. Revista Perspectiva Empresarial, 8(1), 113-126.

Recibido: 23 de enero de 2021 Aceptado: 30 de junio de 2021
RESUMEN Uno de los principales retos para las organizaciones hoy es lograr una óptima integración de los colaboradores al considerar las dificultades implícitas en la diversidad generacional de equipos con distintas motivaciones, estilos de trabajo, expectativas y conductas. Objetivo. Revisar la taxonomía de las generaciones en el ámbito laboral a través de un análisis bibliométrico en el tiempo y desde la perspectiva de distintos países. Metodología. Se analizaron 293 documentos recopilados de las bases de datos Web of Science y Scopus en el período comprendido entre 1957 y 2020; para ello se utilizó el software R, Biblioshiny, con interfaz Bibliometrix. Resultados. Los principales hallazgos revelan un aumento significativo de estudios a través del tiempo, así como el hecho de que las generaciones y los períodos que las determinan tienen una definición heterogénea de acuerdo con el país en el que se estudian. Conclusiones. Para efectos de un mejor y más eficiente diseño de estrategias de capital humano, las organizaciones deben ser conscientes de las diferencias generacionales.

PALABRAS CLAVE capital humano, fuerza laboral multigeneracional, generación $\mathrm{X}$, generación Y, baby boomers.

Trends in the taxonomy of generations in the workplace: a bibliometric analysis
KEYWORDS human capital, multigenerational workforce, generation X, generation $\mathrm{Y}$, baby boomers. 


\section{Tendências na taxonomia das gerações no local de trabalho: uma análise bibliométrica}

RESUMO Um dos principais desafios para as organizações hoje é conseguir uma integração ideal dos funcionários, considerando as dificuldades implícitas na diversidade geracional de equipes com diferentes motivações, estilos de trabalho, expectativas e comportamentos. Objetivo. Revise a taxonomia de gerações no local de trabalho por meio de uma análise bibliométrica ao longo do tempo e da perspectiva de diferentes países. Metodologia. Foram analisados 293 documentos coletados nas bases de dados Web of Science e Scopus no período entre 1957 e 2020; para isso, foi utilizado o software R, Biblioshiny, com interface Bibliometrix. Resultados. As principais descobertas revelam um aumento significativo dos estudos ao longo do tempo, bem como o fato de as gerações e os períodos que as determinam terem uma definição heterogênea de acordo com o país em que são estudadas. Conclusões. Para o propósito de um desenho melhor e mais eficiente das estratégias de capital humano, as organizações devem estar cientes das diferenças geracionais.

PALAVRAS CHAVE capital humano, força de trabalho multigeracional, geração $X$, geração Y, baby boomers. 


\section{Introducción}

Hoy en día, se enfrenta un entorno laboral compuesto de diferentes generaciones en el ámbito laboral con diversas escalas de valores laborales (Cennamo and Gardner, 2008); esto requiere un mayor entendimiento de los valores y actitudes en el trabajo, relacionados con el perfil y la edad de los colaboradores por parte de los equipos de capital humano. La posibilidad de conocer, utilizar y anticipar los estereotipos generacionales permite generar estrategias en los distintos procesos de capital humano como son la atracción, integración, desarrollo, retención y desincorporación de las personas en las organizaciones.

Si bien varios autores marcan períodos detiempo distintos para definir las generaciones, es relevante destacar que en cada país existen variaciones respecto de la mayoría de las investigaciones. Como es el caso de las realizadas en los Estados Unidos, que es el país con la mayor generación de artículos de investigación sobre el tema.

Por lo anterior, este estudio bibliométrico tiene como objetivo revisar la taxonomía de las generaciones en el ámbito laboral a través del tiempo y desde la perspectiva de distintos países. Los hallazgos de esta investigación consisten en un aumento significativo de los estudios a través del tiempo y la confirmación de que las generaciones y los períodos que las determinan tienen una definición heterogénea de acuerdo con el país en el que se estudian.

\section{Materiales y métodos}

Se realizó un análisis bibliométrico del tema de "las generaciones en el ámbito laboral", utilizando una base de datos obtenida el 14 de septiembre de 2020 a partir de una búsqueda de revistas Web of Science (WoS) y Scopus. Las palabras clave utilizadas para la búsqueda en el título fueron "generation" y "organization" o "workforce" o "workplace", el período de búsqueda comprendió entre 1957 y
2020. Se obtuvieron 988 resultados extraídos en archivos BIBy XLS para su procesamiento, los cuales posteriormente fueron depurados para eliminar todos los documentos no relacionados con el tema de investigación; al final, se obtuvo una base total de 293 documentos.

Durante el proceso se utilizó el software Microsoft Excel para hacer arreglos a la base de datos y generar un archivo con extensión CSV para ser utilizado en el software R, Biblioshiny, con interfaz Bibliometrix, con la finalidad de realizar el análisis de datos y su interpretación.

Por medio de este análisis se obtuvo la producción anual de artículos publicados relacionados con las generaciones en el ámbito laboral y el promedio de número de citas por artículo, la lista de los 20 autores y artículos más citados, los países con mayor número de citaciones, las fuentes más citadas en publicaciones relacionadas con el tema, las palabras clave de autor con más recurrencia y su correlación, así como los principales países donde se trata el tema.

\section{Resultados}

Con base en la metodología se presentan los siguientes resultados. En la tabla 1 se observa que el estudio generacional en el ámbito laboral ha sido abordado continuamente a través del tiempo con un número relevante de palabras clave y autores. 
Tabla 1. Información general sobre artículos publicados

\begin{tabular}{|c|c|}
\hline Descripción & Resultados \\
\hline \multicolumn{2}{|l|}{ Información principal sobre los datos } \\
\hline Espacio de tiempo & $1957-2020$ \\
\hline Fuentes (revistas, libros entre otros) & 235 \\
\hline Documentos & 293 \\
\hline Años promedio desde la publicación & 8,16 \\
\hline Citas promedio por documentos & 16,64 \\
\hline Citas promedio por año por documento & 1,592 \\
\hline Referencias & 11864 \\
\hline \multicolumn{2}{|l|}{ Tipos de documentos } \\
\hline Artículo & 293 \\
\hline \multicolumn{2}{|l|}{ Contenido del documento } \\
\hline Palabras clave más (ID) & 552 \\
\hline Palabras clave del autor (DE) & 647 \\
\hline \multicolumn{2}{|l|}{ Autores } \\
\hline Autores & 629 \\
\hline Apariciones del autor & 686 \\
\hline Autor de documentos de un solo autor & 87 \\
\hline Autores de documentos de varios autores & 542 \\
\hline \multicolumn{2}{|l|}{ Colaboración de los autores } \\
\hline Documentos de un solo autor & 94 \\
\hline Documentos por autor & 0,466 \\
\hline Autores por documento & 2,15 \\
\hline Coautores por documento & 2,34 \\
\hline Índice de colaboración & 2,72 \\
\hline
\end{tabular}

Fuente: elaboración propia por parte de los autores.

En la figura 1 se observa una tendencia creciente en la investigación de las generaciones a partir de 2008, ya que previo a este período la publicación de artículos era relativamente estable. En 2008 se presentó un incremento de más del $100 \%$ en el número de artículos y para 2019 se observó que se duplicó el interés en el tema. 


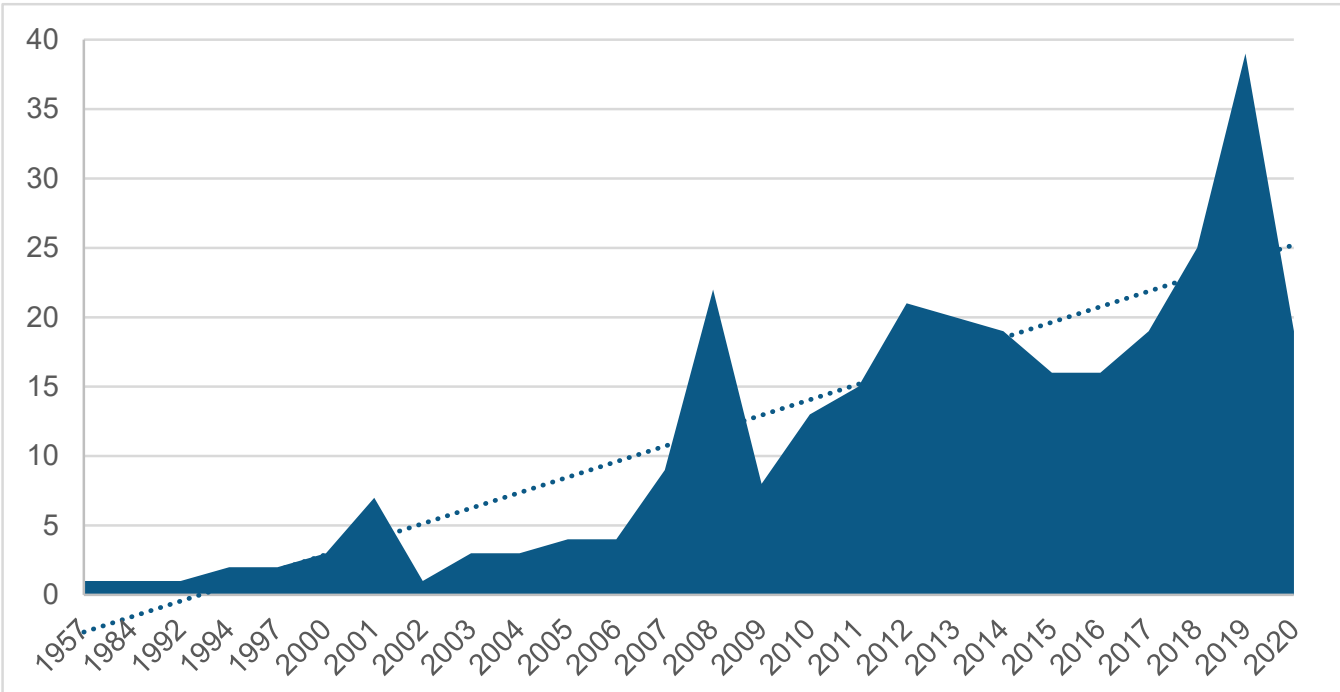

Figura 1. Número de artículos publicados de "las generaciones en el ámbito laboral”. Fuente: elaboración propia por parte de los autores.

No es posible identificar una correlación entre el número de citas y el número de artículos publicados en los últimos 63 años. A pesar de tenerunatendencia creciente en las publicaciones, el número de citas promedio muestra una disminución; esto se puede interpretar como la generación de nuevas ideas y propuestas que no están basadas en investigaciones previas. Al respecto, se afirma que la generación de ideas grupales depende preponderantemente de la creatividad organizacional (Paulus and Yang, 2000).

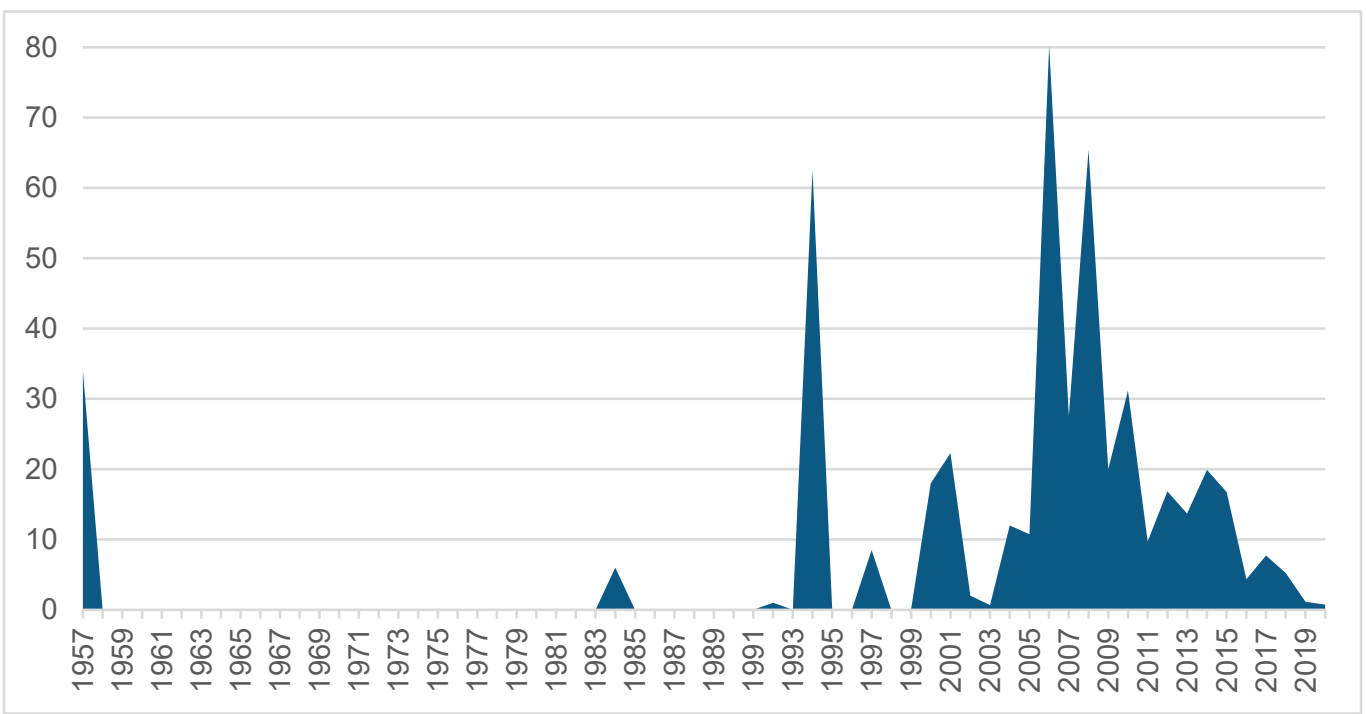

Figura 2. Promedio de número de citas por artículo de "las generaciones en el ámbito laboral”. Fuente: elaboración propia por parte de los autores. 
En la base de datos de información existen 9 artículos que han sido citados en más de 100 ocasiones y los siguientes 10 han sido citados entre 50 y 99 veces (figura 3). Una base de información tan amplia permite identificar los documentos más relevantes en términos de referencia utilizada para la creación de nuevas investigaciones.
Es importante hacer notar que cinco de estos 20 documentos fueron elaborados en la última década y de estos solo uno supera las 200 citaciones (figura 3 ), por lo que es un logro notable haber alcanzado este número de citas en tan solo 5 años.

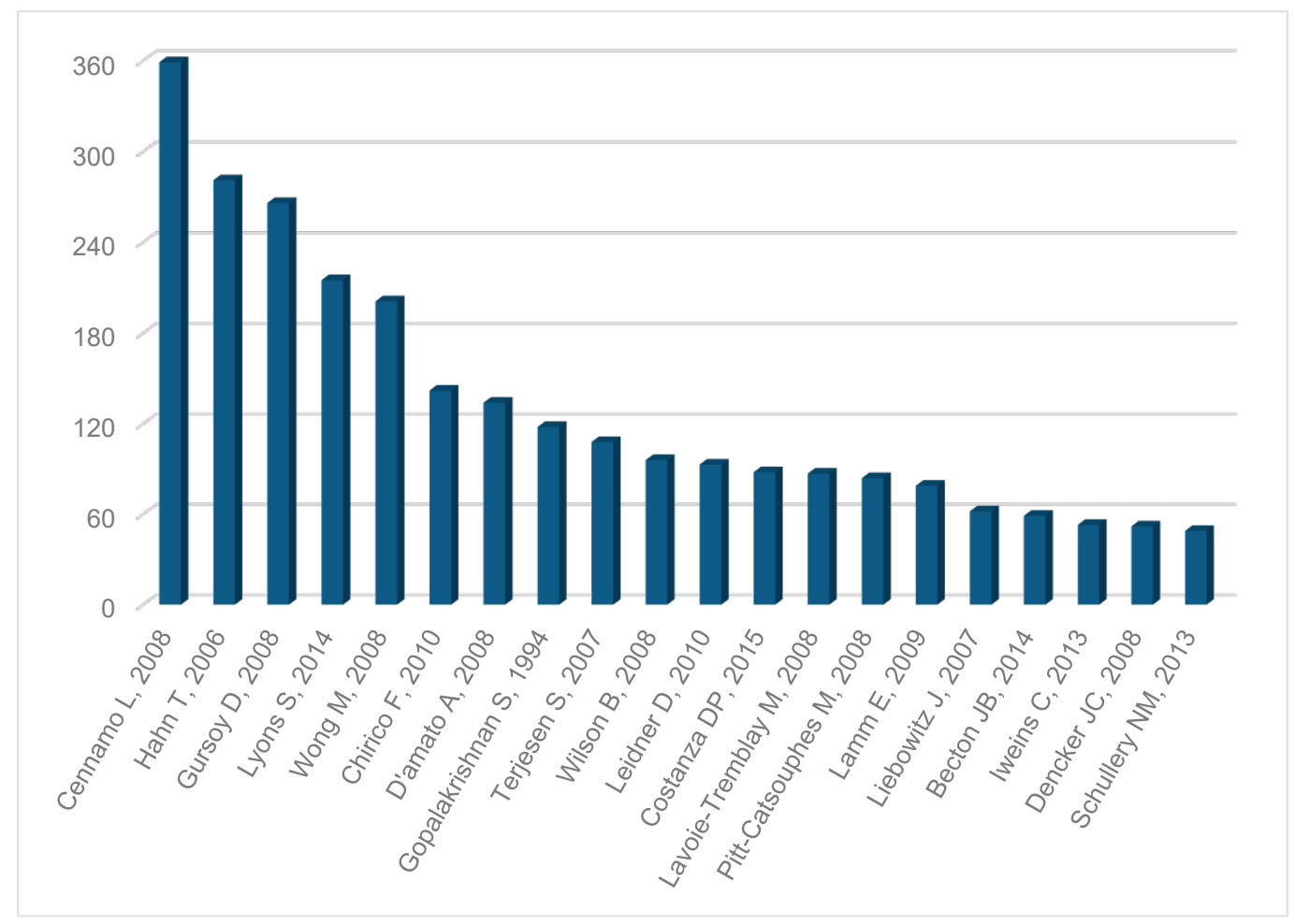

Figura 3. Artículos publicados más citados sobre “las generaciones en el ámbito laboral”. Fuente: elaboración propia por parte de los autores.

Estados Unidos es claramente el país más citado en estudios generacionales, superando casi tres veces al siguiente en la lista (figura 4).

Los 20 países más citados cuentan con un total de 3807 citaciones, de las cuales el $59 \%$ corresponden a Estados Unidos y Canadá. Es relevante identificar que el $80 \%$ de las citaciones pertenecen a países donde el idioma oficial es el inglés. 


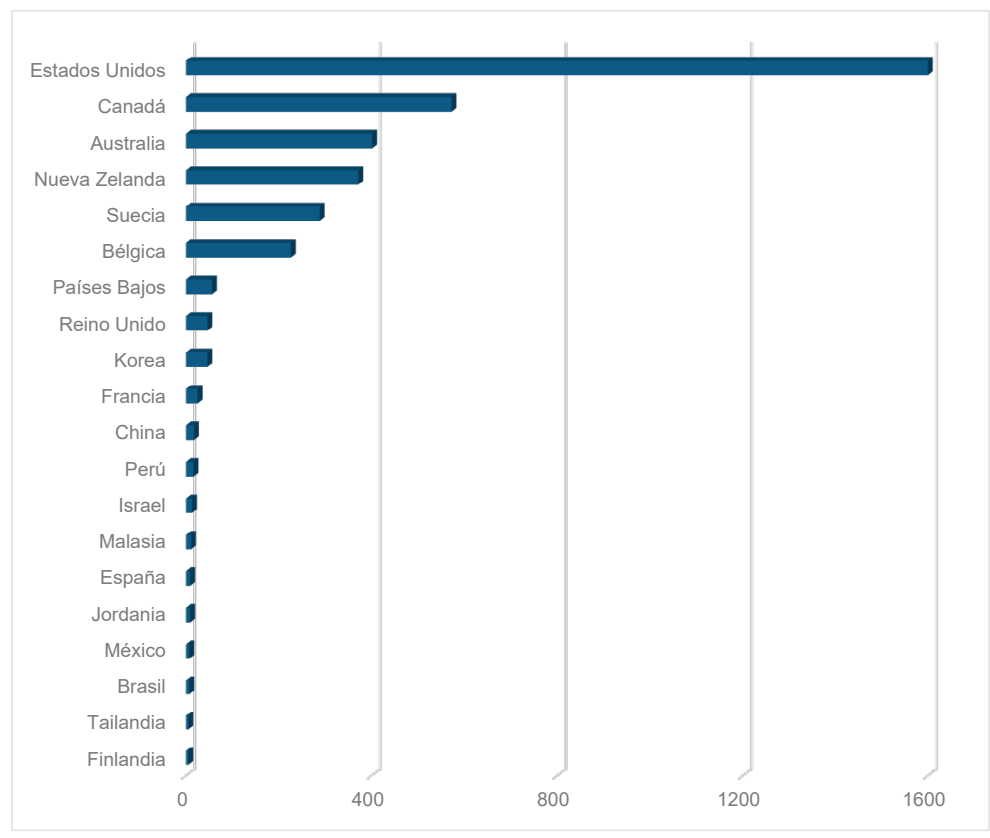

Figura 4. Países con mayor número de citaciones en artículos sobre "las generaciones en el ámbito laboral”. Fuente: elaboración propia por parte de los autores.

Las dos revistas con mayor número de documentos publicados corresponden a publicaciones especializadas en el tema de psicología aplicada y organizacional, pues los rasgos psicológicos son deferentes entre las diferencias generacionales (Twenge and Campbell, 2008;
Gursoy, Maier and Chi, 2008). En el tercer lugar de la lista se encuentra una revista de administración (Academy of Management Journal). Es posible identificar también revistas dedicadas a estudios de temas organizacionales relacionados con los recursos humanos (figura 5).

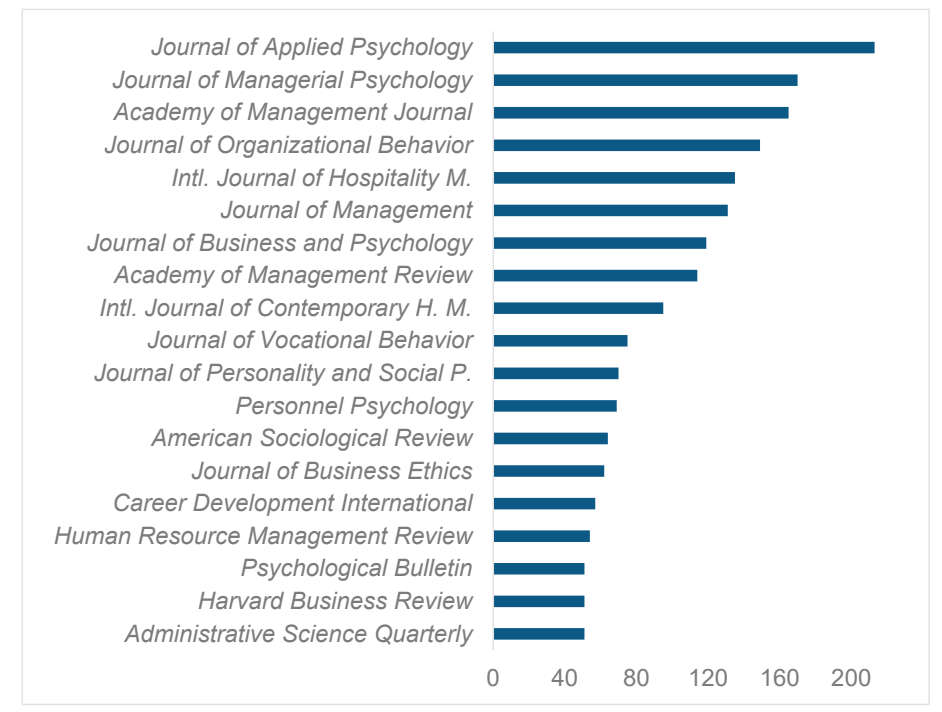

Figura 5. Fuentes más citadas en artículos publicados sobre generaciones en el ámbito laboral. Fuente: elaboración propia por parte de los autores. 
Es interesante identificar que las palabras clave a que se hace referencia en estos artículos muestran una mayor recurrencia sobre la palabra "generación Y"; a la cual le gusta el cumplimiento de las expectativas profesionales según las investigaciones (Kong, Okumus and Bu, 2020); mientras que la "generación X" es la que presenta menor número de ocurrencia entre las clasificaciones de los grupos generacionales (figura 6).
También destaca que la segunda palabra clave más utilizada por los autores de estos artículos está relacionada con las diferencias generacionales y que la palabra "generación Z" no está dentro de las 20 más recurrentes, a pesar de que se ha trabajado en la retención de esta fuerza laboral y su talento (Goh and Okumus, 2020).

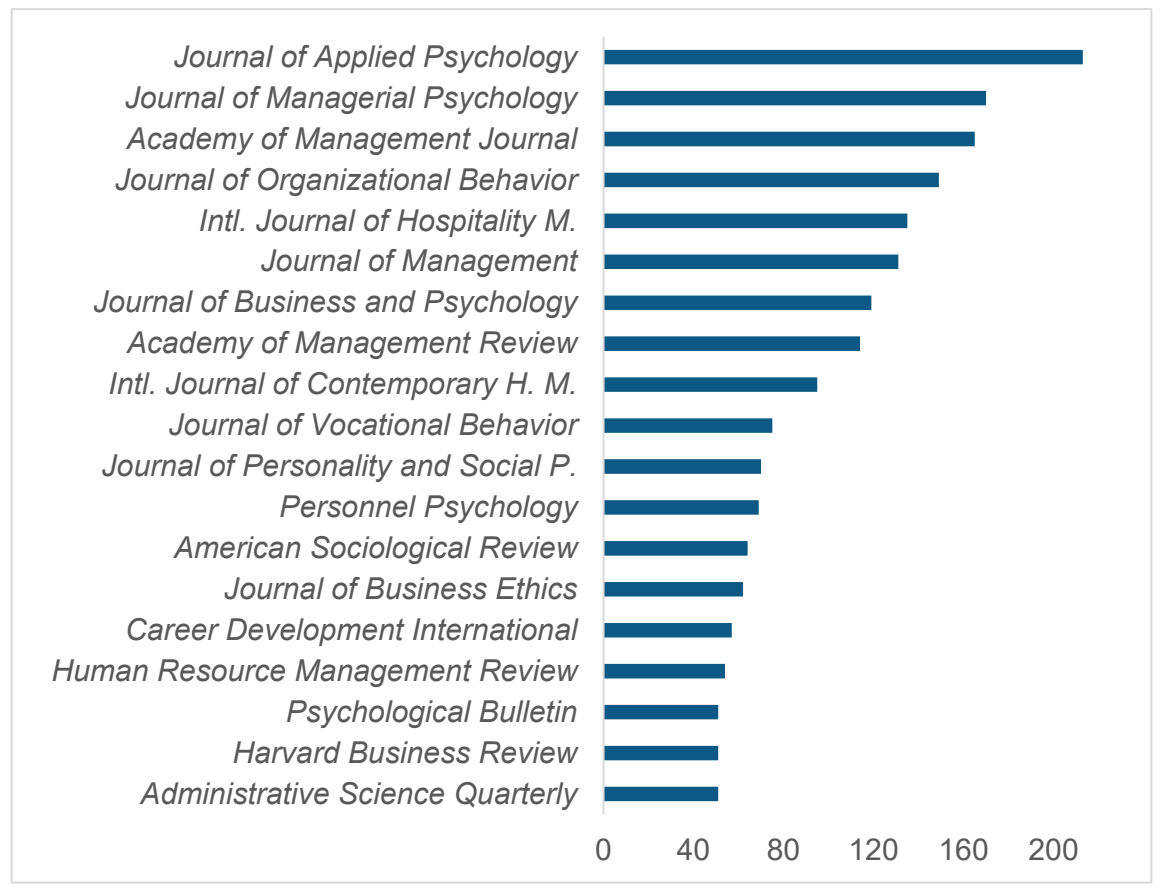

Figura 6. Palabras clave del autor con más recurrencia. Fuente: elaboración propia por parte de los autores.

Por su parte a través del siguiente diagrama es posible identificar las palabras correlacionadas (figura 7). Se observa que las generaciones de millennials, baby boomers y generación $\mathrm{X}$ generalmente se mencionan en el mismo documento. De igual manera cuando se trata el tema de forma más general al hacer referencia a las "generaciones", las palabras mayormente correlacionadas son: retención, liderazgo, compromiso y diversidad generacional.
Se observa una tendencia a analizar de forma particular a la generación Y en conjunto con sus valores y actitudes, incluyendo las diferencias generacionales en el liderazgo y su importancia en el compromiso con los empleados (Stevanin et al., 2020; Jung, Seo and Yoon, 2020). 


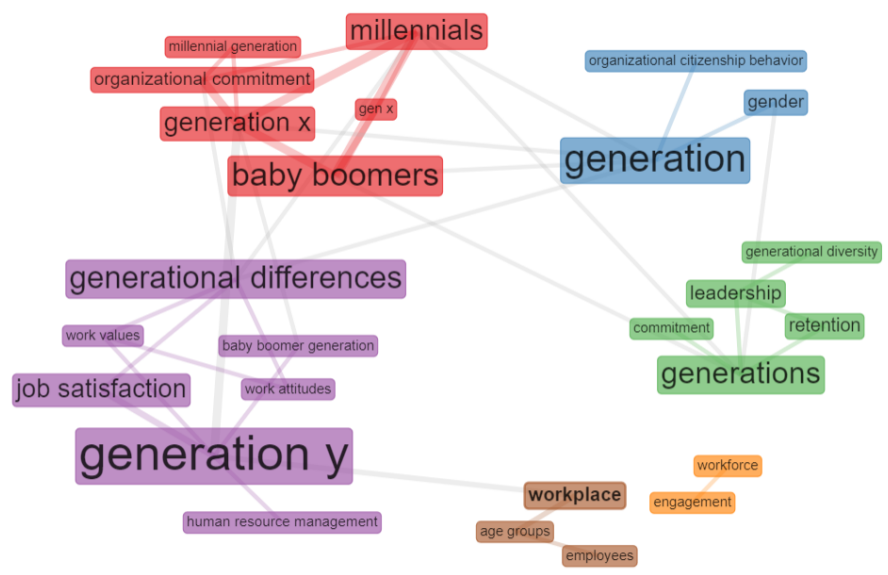

Figura 7. Correlación entre las palabras clave del autor con más recurrencia. Fuente: elaboración propia por parte de los autores.

Se realizó un dendrograma ${ }^{1}$ en el que es posible identificar las palabras baby boomer, millennialgeneration, generation Xy organizational commitment como las palabras raíz de los temas relacionados con las generaciones en el ámbito laboral; derivando posteriormente en conceptos vinculados a capital humano tales como relaciones intergeneracionales, comunicación, compromiso, satisfacción laboral, reclutamiento, retención, diversidad generacional, cultura organizacional entre otros.

Es interesante observar en el dendrograma las relaciones entre palabras clave (figura 8).

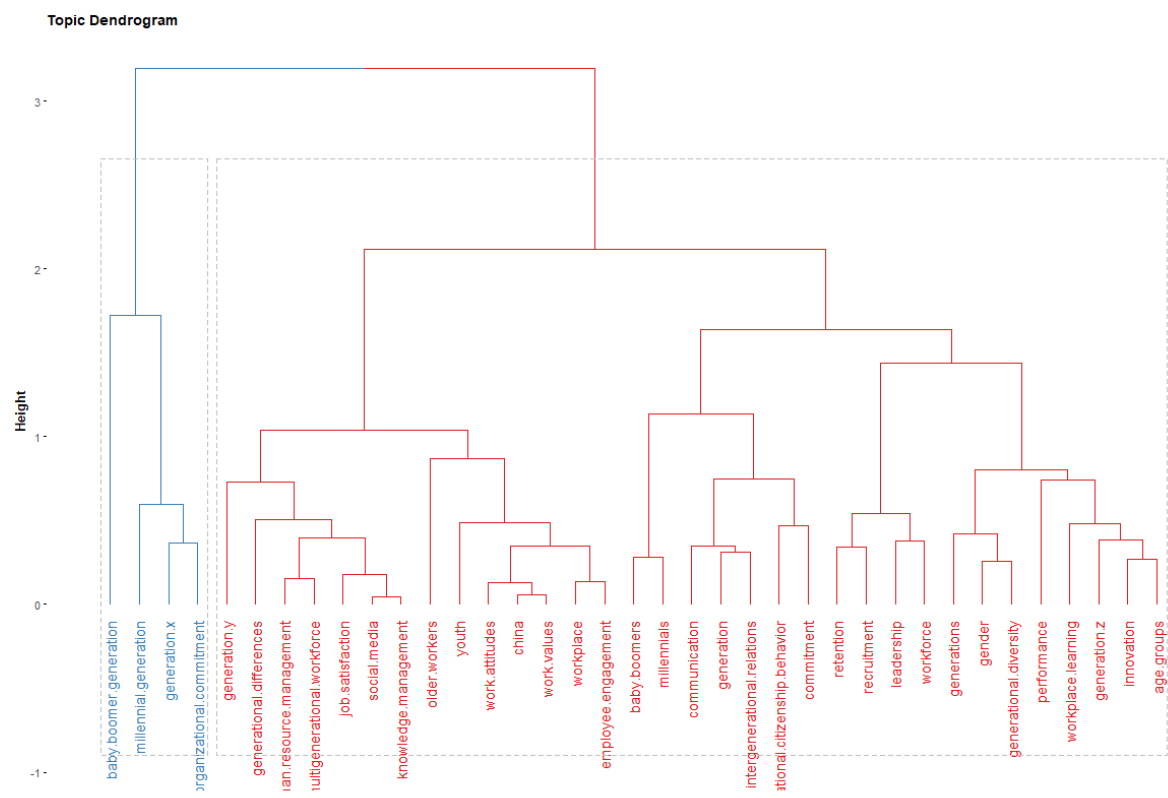

Figura 8. Dendrograma de temas, utilizando palabras clave del autor. Fuente: elaboración propia por parte de los autores.

${ }^{1}$ Un dendrograma es un tipo de representación gráfica o diagrama de datos en forma de árbol que organiza los datos en subcategorías que se van dividiendo en otras hasta llegar al nivel de detalle deseado. 
Por último, el país con mayor investigaciones sobre el tema de generaciones es Estados Unidos; con una colaboración focalizada en países angloparlantes (figura 9).

\section{Contry Collaboration Map}

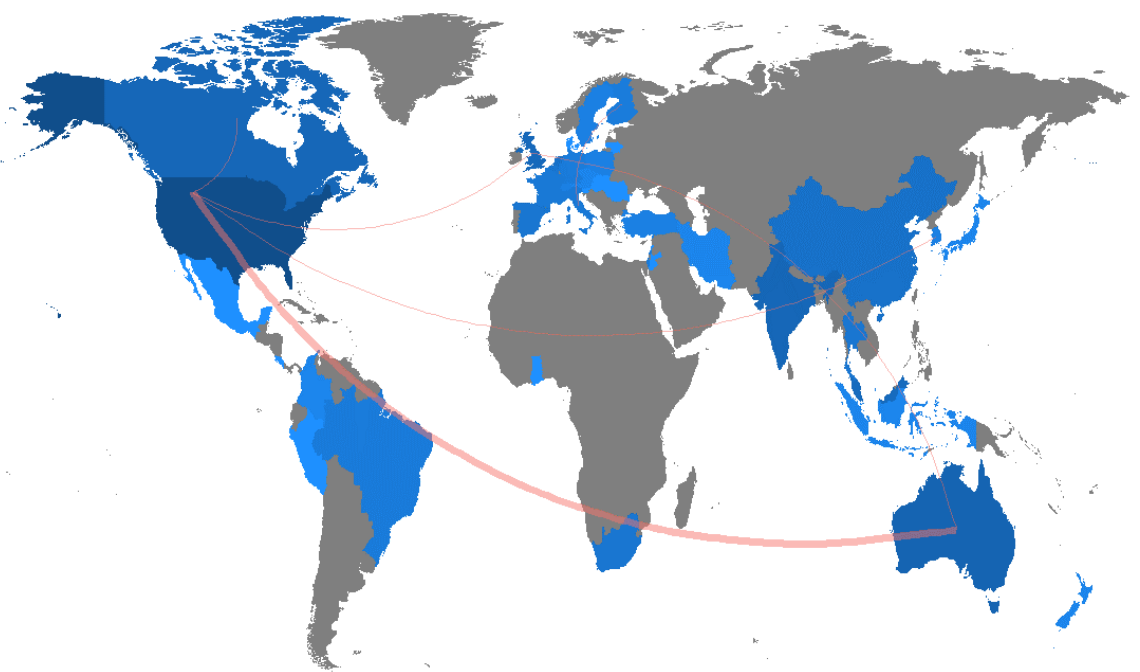

Figura 9. Mapa de colaboración entre países. Fuente: elaboración propia por parte de los autores.

\section{Discusión}

\section{Análisis de las generaciones en el ámbito laboral desde la perspectiva de diferentes países}

Akhavan et al. (2016) identifican grupos generacionales en Irán, utilizando entrevistas y métodos cualitativos que evalúan la opinión colegiada de directivos académicos y profesionales. Dentro de los resultados de su investigación se muestra el resumen de las opiniones de los expertos sobre los grupos generacionales en Irán y se realiza una comparación con grupos generacionales de países occidentales. Los hallazgos que muestran los autores como conclusión es que hay cinco generaciones separadas con diferentes características en Irán.

Por otro lado Park y Park (2018) revisaron la literatura actual sobre cuestiones generacionales en Corea, específicamente cómo se relaciona con el lugar de trabajo. Utilizan varias combinaciones y variaciones de palabras clave tales como brecha generacional, generaciones, baby boomers, generación X, generación Y, millennials y lugar de trabajo multigeneracional para comparar las cuatro generaciones en Corea respecto de las de Estados Unidos. Este artículo concluyó que existen distintas generaciones en el lugar de trabajo coreano.

Por su parte Rani y Samuel (2016) tuvieron como objetivo proporcionar conocimiento de las diferencias generacionales en los valores laborales y el ajuste de valores persona-organización de las tres generaciones de trabajadores en la India; además de explorar la relación entre la persona-organización, intención de desajuste y rotación de la generación Y. Midieron los valores en el trabajo a través de la encuesta sobre valores laborales de Lyons LWVS-, presentando resultados de las diferencias generacionales. Sus conclusiones mostraron que las diferencias en los valores laborales de la generación Y y otras generaciones sugieren que los gerentes deben estar preparados para lidiar con una nueva generación de trabajadores con una gama de valores laborales distintos. De esta forma se destaca el rol de la generación de conocimiento y el aprendizaje social (Berkes, 2009). 
Entretanto Tang, Wang y Zhang (2017) investigan diferencias generacionales en los valores humanos básicos (Schwartz et al., 2012) en el lugar de trabajo chino. Entre los resultados que presenta este documento está el resumen de estudios sobre cohortes generacionales chinos y sus diferencias generacionales, así como la relación que existe entre los valores y las generaciones. Los autores encontraron similitudes en los valores de conservación, automejora y autotrascendencia entre los valores anteriores a la reforma y generaciones posteriores a la reforma. También exploraron cómo los efectos de los valores en el comportamiento de los empleados cambiaron en las generaciones de China.

En Australia se realizó una investigación sobre el "tipo de cambio generacional" que tuvo como objetivo investigar el intercambio de satisfacción de desarrollo profesional por compromiso organizacional y descuido del trabajo en el que la organización del estudio de caso es una organización australiana de investigación del sector público. En los resultados se presentan estadísticas descriptivas y un análisis del compromiso organizacional, abandono del trabajo y satisfacción del desarrollo profesional. La conclusión a la que se llega establece que es más probable quelageneración Xintercambie la satisfacción con el desarrollo profesional por niveles más altos de compromiso organizacional y un menor abandono del trabajo que los boomers (Benson et al., 2018).

Lyons y Schweitzer (2017), en Canadá, elaboraron una investigación que busca una conceptualización más matizada y subjetiva de la generación como base de la identidad social e individual en las organizaciones. Los resultados presentados muestran las razones del grupo analizado para identificarse con un grupo generacional, las razones para no identificarse y las razones para no estar seguros sobre su identidad generacional. Como conclusión sugieren que las personas hacen uso de la generación como categoría social, pero algunos no se identifican con ningún grupo y otros no están seguros de su identificación.

Un artículo elaborado en Finlandia presenta como propósito resaltar y analizar críticamente cuestiones relacionadas con la edad y la generación y sus intersecciones con otras divisiones sociales en la vida laboral y su importancia para la forma de trabajar, de construir carreras, organizaciones e innovaciones laborales relacionadas (Hahn et al., 2006). Al utilizar datos cualitativos de nueve organizaciones en Finlandia, los resultados se relacionan con la forma en que se da en las organizaciones un discurso sobre la edad y la generación. Así como el hecho de que el género alguna vez fue en gran parte invisible en los estudios de organización. En conclusión, parece que la edad y la generación a menudo no se articulan completamente en el análisis o se presentan a través de conceptos supuestamente más 'neutrales' como cohortes o sucesión (Niemistö, Hearn and Jyrkinen, 2016).

Con el propósito de ofrecer información sobre los estereotipos que las generaciones tienen de su propia generación y de otras, un artículo de investigación en 2019 basado en una muestra compuesta por ciudadanos de habla holandesa en Bélgica presenta en sus resultados mapas generacionales cognitivos de dosy tres dimensiones para las generaciones X y Y. Su conclusión es que la investigación demuestra que las características generacionales están vivas y bien, por lo menos en la mente de los gerentes (Van Rossem, 2019).

Comunicación de los empleados, participación laboral y compromiso organizacional son los temas que se estudian para los miembros de la generación millennial en los Estados Unidos en el artículo de Walden, Jung y Westerman (2017). Su propósito de estudio fue explorar la relación entre el trabajo compromiso y dos componentes clave de las relaciones empleado-organización. Entre los resultados publicados están el resumen de información demográfica ( $\mathrm{N}=539)$, medidas de la encuesta, la correlación de orden cero entre todas las variablesy fiabilidad, los efectos indirectos significativos delos predictores a través de variables intervinientes y los predictores del compromiso de los empleados. Los autores concluyen que cuando los empleados de la generación millennial están comprometidos con su trabajo, su compromiso con la organización se refuerza y la probabilidad de que abandonen la organización disminuye (Walden, Jung and Westerman, 2017).

Por último, Montaudon-Tomas et al. (2019) muestran en su investigación un ejemplo de equipos de alto desempeño en una fuerza laboral multigeneracional en el ámbito académico en 
México. En la descripción del caso práctico se plantea la forma en que se desarrollaron los equipos como parte de la planificación estratégica de una escuela de negocios mexicana. Como conclusión, se determina que la percepción de desempeño del equipo tenía una correlación positiva con su evaluación de productividad y que la calificación del equipo sobre el valor del trabajo multigeneracional está relacionada con su productividady/o la percepción de desempeño de los miembros del equipo.

Al considerar estos resultados es posible identificar que el tema de las generaciones en el ámbito laboral es un tema de interés e investigación para muchos países independientemente de su idioma o región geográfica, coincidiendo en que las generaciones no pueden ser generalizadas a nivel global.

\section{Conclusiones}

Este estudio tuvo como objetivo revisar la taxonomía de las generaciones en el ámbito laboral a través del tiempo y desde la perspectiva de distintos países. Específicamente, investigar si las generaciones y los períodos que las determinan tienen una definición heterogénea de acuerdo con el país en el que se estudian. Los resultados muestran: (i) un aumento significativo en la actividad de investigación de este tema en los últimos años, probablemente debido a los marcados cambios históricos que se han dado de forma global; (ii) que a pesar de que existe una preferencia muy marcada en el número de ocasiones en que se cita como país a Estados Unidos en este tema, también existe interés en otros países; (iii) definiciones heterogéneas de las generaciones en lo que corresponde a los períodos de clasificación e incluso en el número de generaciones en períodos de tiempo iguales.

Para efectos de un mejor y más eficiente diseño de estrategias de capital humano, las organizaciones deben ser conscientes de las diferencias generacionales al tomar en cuenta que el concepto de generación se define como "un grupo identificable que comparte un período de nacimiento y eventos de vida significativos ocurridos en etapas críticas de sus vidas" (Kupperschmidt, 2000, p. 66); de tal manera que la clasificación de cada país puede ser distinta a la realizada por Estados Unidos, aunque esta sea la más publicada.

Es posible que las investigaciones futuras puedan enfocarse a establecer un proceso que permita definir: (i) las generaciones para cada país en función de los cambios en sus respectivos entornos y (ii) las características que las diferencian.

La principal limitación que se debe reconocer es que a pesar de que existe una abundante investigación sobre las diferencias generacionales en el ámbito laboral, frecuentemente carecen de rigory varios autores establecen que hace falta más investigación empírica para validar las diferencias entre las generaciones.

\section{Referencias}

Akhavan, A.R. et al. (2016). The impact of generational groups on organizational behavior in Iran. Human Systems Management, 35(3), 175-183.

Benson, J. etal. (2018). The generational "exchange" rate: How generations convert career development satisfaction into organisational commitment or neglect of work. Human Resource ManagementJournal,28(4), 524-539.

Berkes, F. (2009). Evolution of co-management: role of knowledge generation, bridging organizations and social learning. Journal of Environmental Management, 90(5), 1692-1702.

Cennamo, L. and Gardner, D. (2008). Generational differences in work values, outcomes and person-organisation values fit. Journal of Managerial Psychology, 23(8), 891-906.

Goh, E. and Okumus, F. (2020). Avoiding the hospitality workforce bubble: Strategies to attract and retain generation $\mathrm{Z}$ talent in the hospitality workforce. Tourism Management Perspectives, 33, 100603.

Gursoy, D., Maier, T.A. and Chi, C.G. (2008). Generational differences: An examination 
of work values and generational gaps in the hospitality workforce. International Journal of Hospitality Management, 27(3), 448-458.

Hahn, T. et al. (2006). Trust-building, knowledge generation and organizational innovations: the role of a bridging organization for adaptive comanagement of a wetland landscape around Kristianstad, Sweden. Human Ecology, 34(4), 573-592.

Jung, H.S., Seo, K.H. and Yoon, H.H. (2020). The importance of leader integrity on family restaurant employees' engagement and organizational citizenship behaviors: Exploring sustainability of employees' generational differences. Sustainability, 12(6), 2504.

Kong, H., Okumus, F. and Bu, N. (2020). Linking organizational career management with Generation Y employees' organizational identity: The mediating effect of meeting career expectations. Journal of Hospitality Marketing \& Management, 29(2), 164-181.

Kupperschmidt, B.R. (2000). Multigenerational employees: Strategies for effective management. The Health Care Manager, 19(1), 65-76.

Lyons, S.T. and Schweitzer, L. (2017). A qualitative exploration of generational identity: Making sense of young and old in the context of today's workplace. Work, Aging and Retirement, 3(2), 209-224.

Montaudon-Tomas, C.M. et al. (2020). High Performance Teams in a Multigenerational Workforce: An Example from Academia in Mexico. En Montaudon-Tomas, C.M. et al. (Eds.), Global Applications of Multigenerational Managementand Leadership in the Transcultural Era (pp. 76-107). Pennsylvania, USA: IGI Global.

Niemistö, C., Hearn, J. and Jyrkinen, M. (2016). Age and generations in everyday organisational life: Neglected intersections in studying organizations. International Journal of Work Innovation, 1(4), 353-374.

Park, S. and Park, S. (2018). Exploring the generation gap in the workplace in South Korea. Human
Resource Development International, 21(3), 276-283.

Paulus, P.B. and Yang, H.C. (2000). Idea generation in groups: A basis for creativity in organizations. Organizational Behavior and Human Decision Processes, 82(1), 76-87.

Rani, N. and Samuel, A. (2016). A study on generational differences in work values and person-organization fit and its effect on turnover intention of Generation Y in India. Management Research Review, 39(12), 16951719.

Schwartz, S. et al. (2012). Refining the theory of basic individual values. Journal of Personality and Social Psychology, 103(4), 663-688.

Stevanin, S. et al. (2020). Nurses' generational differences related to workplace and leadership in two European countries. Western Journal of Nursing Research, 42(1), 14-23.

Tang, N., Wang, Y. and Zhang, K. (2017). Values of Chinese generation cohorts: Do they matter in the workplace? Organizational Behavior and Human Decision Processes, 143, 8-22.

Twenge, J.M. and Campbell, S.M. (2008). Generational differences in psychological traits and their impact on the workplace. Journal of Managerial Psychology, 23(8), 862-877.

Van Rossem, A.H.D. (2019). Generations as social categories: An exploratory cognitive study of generational identity and generational stereotypes in a multigenerational workforce. Journal of Organizational Behavior, 40(4), 434-455.

Walden, J., Jung, E.H. and Westerman, C.Y.K. (2017). Employee communication, job engagement, and organizational commitment: A study of members of the Millennial Generation. Journal of Public Relations Research, 29(2-3), 73-89. 